\title{
In situ observations of a magnetosheath high-speed jet triggering magnetopause reconnection
}

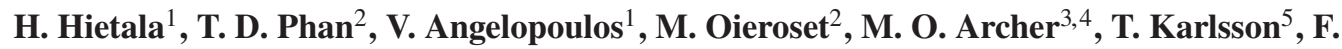 \\ Plaschke $^{6,7}$ \\ ${ }^{1}$ Dept. of Earth, Planetary, and Space Sciences, University of California, Los Angeles, USA \\ ${ }^{2}$ Space Science Laboratory, University of California, Berkeley, USA \\ ${ }^{3}$ School of Physics and Astronomy, Queen Mary University of London, London, UK \\ ${ }^{4}$ The Blackett Laboratory, Imperial College London, UK \\ ${ }^{5}$ Space and Plasma Physics, School of Electrical Engineering, KTH Royal Institute of Technology, Stockholm, Sweden \\ ${ }^{6}$ Space Research Institute, Austrian Academy of Sciences, Graz, Austria \\ ${ }^{7}$ Institute of Physics, University of Graz, Graz, Austria
}

\section{Key Points:}

- THEMIS probes (string-of-pearls formation) recorded magnetopause in-and-out motion during jet impact

- No reconnection flows for the pre-impact crossing; 3 probes observed reconnection after the impact

- Compression by the high pressure jet initiated reconnection in an originally thick magnetopause 


\begin{abstract}
Magnetosheath high-speed jets-localized dynamic pressure enhancements typically of $\sim 1$ Earth radius in size-impact the dayside magnetopause several times per hour. Here we present the first in situ measurements suggesting that such an impact triggered magnetopause reconnection. We use observations from the five THEMIS spacecraft in a stringof-pearls configuration on August 7, 2007. The spacecraft recorded magnetopause in-andout motion during an impact of a magnetosheath jet $\left(V_{N} \sim-300 \mathrm{~km} / \mathrm{s}\right.$ along the magnetopause normal direction). There was no evidence for reconnection for the pre-impact crossing, yet three probes observed reconnection after the impact. We infer that the jet impact compressed the originally thick $\left(60-70 d_{\mathrm{i}}\right)$, high magnetic shear $\left(140-160^{\circ}\right)$ magnetopause until it was thin enough for reconnection to occur. Magnetosheath high-speed jets could therefore act as a driver for bursty dayside reconnection.
\end{abstract}

\title{
1 Introduction
}

The processes taking place within the foreshock, bow shock, and magnetosheath regions affect the inhomogeneous plasma and energy flow in contact with the outer boundary of the magnetosphere, the magnetopause. Magnetosheath high-speed jets (HSJs; e.g., Nemecek et al. [1998]; Savin et al. [2008]; Hietala et al. [2009]) are some of the most prominent transient phenomena on the dayside: the jets are localized enhancements of dynamic pressure with typical scales of about one Earth radius $\left(R_{\mathrm{E}}\right)$ [e.g., Plaschke et al., 2016], where the dynamic pressure can be increased up to a factor of 4 compared to the solar wind [Plaschke et al., 2013], and by a factor of 15 compared to the ambient magnetosheath [Archer and Horbury, 2013]. Statistical studies show that most subsolar HSJs occur during intervals of stable radial interplanetary magnetic field (IMF) [Plaschke et al., 2013; Archer and Horbury, 2013]. HSJs are thus thought to be connected to the quasiparallel bow shock geometry and the ion foreshock processes. For such cases, Hietala et al. [2009] proposed a formation mechanism based on intrinsic quasi-parallel bow shock ripples [e.g., Schwartz and Burgess, 1991; Blanco-Cano et al., 2009]: Solar wind plasma entering the magnetosheath through locally inclined shock surfaces (at the ripples) will be less thermalized, i.e., faster than the ambient plasma, constituting the jets seen in the magnetosheath. Comparison of analytic modeling and HSJ statistics [Hietala and Plaschke, 2013], as well as recent hybrid simulations [Karimabadi et al., 2014; Omidi et al., 2016; Hao et al., 2016] support this idea. A minority of jets appears to be formed due to IMF discontinuities [Lin et al., 1996a,b; Archer et al., 2012].

Plaschke et al. [2016] estimated that jets larger than $2 R_{\mathrm{E}}$ in diameter perpendicular to the flow impact the magnetopause many times per hour. When HSJs hit the magnetopause, they may cause large amplitude $\left(\sim 1 R_{\mathrm{E}}\right.$ deep) yet localized boundary indentations [Shue et al., 2009; Amata et al., 2011; Hietala et al., 2012; Dmitriev and Suvorova, 2012; Archer et al., 2012]. Their magnetospheric consequences include generating boundary surface waves, poloidal and compressional waves, and field-line resonances [Plaschke et al., 2009a; Archer et al., 2013a,b], which may in turn affect radiation belt electrons [e.g., Elkington et al., 2003; Turner et al., 2012].

Could HSJ impacts also affect local magnetopause reconnection? It is natural to expect that, since the jets may change the local conditions for reconnection at least in two ways: (i) Their high pressure impact may reduce the magnetopause current sheet thickness, hence triggering reconnection. (ii) They may change the shear angle between the magnetospheric and magnetosheath magnetic field lines, hence affecting the $\Delta \beta$-shear relation [Swisdak et al., 2010; Phan et al., 2013] that predicts whether or not asymmetric reconnection is suppressed by diamagnetic drift. The change in magnetic shear can be due to the magnetic field orientation within the jets (i.e., on the magnetosheath side). It can also be due to the magnetopause indentation caused by the jet's dynamic pressure, 
as the orientation of the magnetospheric field lines gets perturbed [Karlsson et al., 2012]. Changes in the magnetic shear angle may turn reconnection on or off.

There are several pieces of evidence suggesting magnetopause reconnection in association with magnetosheath high-speed jets: observations of penetration of magnetosheath particles into the magnetosphere [Gunell et al., 2012, 2014; Dmitriev and Suvorova, 2015], and of ionospheric flows and particle precipitation during jet impacts [Hietala et al., 2012]. Recently, Han et al. [2017] found dayside diffuse aurora showing discrete north-south aligned arcs, which were associated with precipitating magnetosheath particles and open field lines (i.e., magnetopause reconnection), magnetopause indentations, and low IMF cone-angle conditions. They suggested that these 'throat aurorae' could be the auroral signatures of HSJ impacts causing magnetopause reconnection.

Here we present the first in situ observations suggesting that a magnetosheath jet triggered magnetopause reconnection. We consider a HSJ impact that was part of a series of jets observed by THEMIS [Angelopoulos, 2008] in a string-of-pearls configuration on August 7, 2007. While various magnetosheath fluctuations may change the $\Delta \beta$-shear relation, we focus on the HSJs' ability to compress the magnetopause current layer: We consider a case where the pre-impact magnetopause, despite favorable $\Delta \beta$ and magnetic shear conditions, was too thick for reconnection to occur.

\section{Data and Methods}

We analyze magnetic field data from the Flux Gate Magnetometers (FGM; [Auster et al., 2008]), plasma data from the Electrostatic Analyzers (ESA; [McFadden et al., 2008]) and the Solid State Telescopes (SST; [Angelopoulos, 2008]), and the electric field measurements from the Electric Field Instrument (EFI; [Bonnell et al., 2008]). We reconstructed the 3D electric field using the assumption $\mathbf{E} \cdot \mathbf{B}=0$ for THC, D, and E. (EFI measurements were not available for THA and B this early in the mission.) The plasma data were calibrated using spacecraft potential estimates derived from the measured electron distributions. We combined the ESA and SST ion measurements to obtain a more complete energy coverage. We use the Geocentric Solar Magnetospheric (GSM) coordinate system.

For the analysis of each of the magnetopause crossings we use the hybrid minimum variance analysis (HMVA; Gosling and Phan [2013]) to obtain the current sheet normal coordinates: The normal direction was determined from $N=B_{1} \times B_{2} /\left|B_{1} \times B_{2}\right|$, where $B_{1}$ and $B_{2}$ are the fields at the two edges of the current sheet. The $M$ (approximately the $\mathrm{X}$-line) direction is from $L^{\prime} \times N$, where $L^{\prime}$ is the maximum variance direction from the minimum variance of the magnetic field [Sonnerup and Cahill, 1967]. Finally, $L=N \times M$. (HMVA method was used because MVA results were not good in terms of eigenvalue ratios and visual inspection.) We employed multi-spacecraft timing to the crossings of the magnetopause magnetospheric edge, the magnetosheath edge, and the point where the maximum variance component $B_{L}=0$. This gave us estimates of the current sheet normal velocities, which we also compared to the Faraday Residue method [Khrabrov and Sonnerup, 1998]. The values for each spacecraft and crossing are given in Table S1 of the Supplementary material.

\section{Results}

\subsection{Event Overview}

The event under investigation was part of a 2-hour interval on August 7, 2007. The interval had several HSJs, identified by requiring that the magnetosheath dynamic pres-

sure $P_{\mathrm{dyn}, X}=\rho V_{X}^{2}$ exceeds half the solar wind dynamic pressure (similar to Plaschke et al. [2013]). Some of the jets have been listed in Dmitriev and Suvorova [2015], but none have 
been previously examined for magnetic reconnection. During this 2-hour interval the solar wind speed was quite high, $\sim 600 \mathrm{~km} / \mathrm{s}$, while the density was $\sim 3 \mathrm{~cm}^{-3}$. The IMF magnitude was $\sim 5 \mathrm{nT}$, but its orientation was not stable.

Figure 1 shows an overview of the HSJ impact of interest at 22:58 UT. The OMNI IMF data is displayed in the top panel (time-shifted by 5 minutes to account for propagation from the bow shock to the magnetopause): the mean $B_{X}$ was $\sim 2.7 \mathrm{nT}$, the $B_{Z}$ was $\sim 2.4 \mathrm{nT}$ (northward), and the $B_{Y}$ changed from close to zero to $\sim 1 \mathrm{nT}$ (clock-angle $\sim 22^{\circ}$ ). The IMF cone-angle was $\sim 43^{\circ}$ (between $36^{\circ}$ and $55^{\circ}$ ), i.e., favorable for HSJ formation [e.g., Plaschke et al., 2013].

The magnetosheath density was $\sim 14 \mathrm{~cm}^{-3}$ (Figure 1(c)), with a corresponding ion inertial length of $d_{\mathrm{i}} \sim 61 \mathrm{~km}$. The five THEMIS spacecraft were dawnwards of the subsolar point, at $\sim 10 \mathrm{~h} \mathrm{MLT} \mathrm{(Figure} 1(1, \mathrm{~m})$ ). THB was the outermost probe and THA the innermost, with THC, D, and $\mathrm{E}$ in a cluster between them. The distance between THD and $\mathrm{E}$ was $\sim 0.19 R_{\mathrm{E}} \sim 20 d_{\mathrm{i}}$, and the distance between $\mathrm{C}$ and $\mathrm{D}$ a mere $\sim 0.07 R_{\mathrm{E}} \sim 7.4 d_{\mathrm{i}}$.

The event began by the magnetopause moving inwards past THB to a location slightly Earthwards of THE. In the magnetosheath THB observed a HSJ (Figure 1(b)) with a density increase (Figure 1(c)) and a large Earthward velocity (Figure 1(d)). Closer to the magnetopause, THC observed a similar density increase (Figure 1(f)), but not the enhanced flow (Figure 1(h)). After the HSJ was observed, the magnetopause moved back out. As shown in detail in the following subsections, at the outbound crossing (before the HSJ was observed), there is no evidence for reconnection outflows at the four probes (e.g., Figure 1(e,h,k)). At the inbound crossing (after the HSJ was observed), there were clear reconnection outflows of $V_{Z} \sim-200 \mathrm{~km} / \mathrm{s}$ (THB, C, D), suggesting that reconnection was triggered by the HSJ impact.

\subsection{Magnetopause Characteristics}

\subsubsection{Outbound Crossing}

Figure 2 shows the outbound magnetopause crossing for THB, C, and E, with the vector quantities shown in the HMVA coordinates of each spacecraft. (THD observations, which are similar to THC due to their small separation, are shown in Figure S1 of the Supplementary material.) To identify the magnetospheric edge of the magnetopause, we use the maximum of the normal electric field $+E_{N}$ for THC, D, and E (Figure 2(e); the magnetospheric Sunward-pointing Hall electric field dominates for asymmetric boundary conditions [e.g., Cassak and Fuselier, 2016; Phan et al., 2016]). This $+E_{N}$ maximum also corresponds well to the end of counter-streaming $0.8-2 \mathrm{keV}$ electrons (Figure $2(\mathrm{~g})$ ). The same transition in electrons from closed to open field lines can be seen at THB. At THE the magnetopause appears to have moved slightly back-and-forth, resulting in two $+E_{N}$ maxima and a short reappearance of counter-streaming electrons.

As the magnetopause was moving Earthward its normal was rather similar for all the probes - mainly in the $X_{\mathrm{GSE}}$ direction with a negative $Y_{\mathrm{GSE}}$ component. The magnetopause normal velocity $v_{N}$ was $\sim 35 \mathrm{~km} / \mathrm{s}$ (determined by multi-spacecraft timing; see Section 2). Its thickness (crossing duration multiplied by $v_{N}$ ) was $\sim 60-70 d_{\mathrm{i}}$. The magnetic shear was $\sim 160^{\circ}$ at $\mathrm{THB}, \sim 150^{\circ}$ at THC and $\mathrm{D}$, and $\sim 140^{\circ}$ at THE. Plasma beta was $\sim 0.1$ in the magnetosphere and $\sim 1-10$ in the magnetosheath (Figure $2(\mathrm{j})$ ).

There were no reconnection outflows ( $L$ direction) within the magnetopause layer (Figure 2(f)). According to the $\Delta \beta$-shear relation [Swisdak et al., 2010; Phan et al., 2013], the magnetosheath $\beta$ was not large enough to suppress reconnection. The (unlikely) scenario that the absence of outflows would be due to all four spacecraft being close to the X-line is excluded by two facts: (a) the magnetopause's large, tens of $d_{\mathrm{i}}$ thickness; and (b) the lack of signatures of finite ion Larmor radius effects [Phan et al., 2016]—-there is 
no negative $E_{N}$ on the magnetospheric side of the magnetopause (Figure 2(e)), nor an associated $-V_{M}$ flow (Figure 2(f)). We conclude that despite the large magnetic shear, the magnetopause was probably too thick to reconnect.

\subsubsection{Inbound Crossing}

Figure 3 shows the inbound magnetopause crossing for THE, C, and B in the HMVA coordinates of each spacecraft. (THD observations, which are similar to THC due to their small separation, are shown in Figure S1 of the Supplementary material.) While for these crossings the positive maxima of the $E_{N}$ are located close to the region where $B_{L}=0$, the magnetospheric edge of the magnetopause can be identified by the sharp change in the $B_{L}$ component (Figure 3(d)), which also coincides with the transition to counter-streaming electrons (Figure 3(g)). (Note that THE spent less than $20 \mathrm{~s}$ in the magnetosheath.)

The magnetopause normal was mainly in the $-Y_{\mathrm{GSM}}$ direction when it crossed THE, $\mathrm{D}$, and $\mathrm{C}$, but at THB it had a more typical $X_{\mathrm{GSM}}$-dominated orientation (see also sketch in Figure 1(1)). Its normal velocity was $\sim 90 \mathrm{~km} / \mathrm{s}$, and thickness $\sim 40-50 d_{\mathrm{i}}$. The magnetic shear was $\sim 100^{\circ}$ at THE, $\sim 130^{\circ}$ at THC and D, and $\sim 155^{\circ}$ at THB. Plasma beta was $\sim 0.15$ in the magnetosphere and $\sim 2-10$ in the magnetosheath (Figure 3(j)).

There is no clear reconnection outflow for THE (Figure 3(f)). For THC and D, however, there is an outflow of $\Delta V_{L} \sim-110 \mathrm{~km} / \mathrm{s}$ below the average magnetosheath flow (THC dotted line). At THB the reconnection outflow is even more clear, $V_{L} \sim-260 \mathrm{~km} / \mathrm{s}$. This is $64 \%$ of the asymmetric Alfvén speed [Cassak and Shay, 2007] $V_{\mathrm{AL} \text {, asym }}=\left(B_{1 L} B_{2 L}\left(B_{1 L^{+}}+\right.\right.$ $\left.\left.B_{2 L}\right) /\left(\mu_{0}\left(\rho_{1} B_{2 L}+\rho_{2} B_{1 L}\right)\right)\right)^{1 / 2} \sim 404 \mathrm{~km} / \mathrm{s}$ corresponding to the THB crossing - a rather typical fraction for magnetopause observations [e.g., Sonnerup et al., 1981; Paschmann et al., 1986; Gosling et al., 1990; Phan et al., 1996; Vines et al., 2015].

\subsection{Magnetosheath Jet Characteristics}

THB observed the magnetosheath high-speed jet for $\sim 40 \mathrm{~s}$, with a peak density of $\sim 26 \mathrm{~cm}^{-3}$ (Figure 1(c)). The ion temperature inside the jet was isotropic, in contrast to the $T_{\mathrm{i} \perp}>T_{\mathrm{i} \|}$ ambient magnetosheath (Figure 2(i)); this is common for HSJs [e.g., Plaschke et al., 2013]. The HSJ velocity was dominated by $V_{N} \sim-300 \mathrm{~km} / \mathrm{s}$ (towards the magnetopause) and $V_{L} \sim-200 \mathrm{~km} / \mathrm{s}$, in both outbound and inbound THB HMVA coordinates (Figures 2(f) and 3(f)). The HSJ dynamic pressure along the magnetopause normal was $P_{\mathrm{dyn}, N} \sim 3 \mathrm{nPa}$, and its total pressure (thermal, magnetic, and dynamic) $P_{\mathrm{tot}, N} \sim 4.5 \mathrm{nPa}$ compared to the ambient magnetosheath $P_{\mathrm{tot}, \mathrm{MSH}, N} \sim 1.4 \mathrm{nPa}$ (not shown).

Closer to Earth, THC observed a $\sim 15 \mathrm{~s}$ density increase to $\sim 22 \mathrm{~cm}^{-3}$ (Figure 1(f)). However, at THC the ions were anisotropic (Figure 2(i)), and there is no clear flow enhancement. In addition, there were no flow or density enhancements, or changes in ion temperature anisotropy, at THD or E. The exact reason is unclear, but it could be due to the HSJ braking as it meets the magnetopause, or the spacecraft being very near the edge of the jet. While the THEMIS string-of-pearls configuration does not allow us to estimate the HSJ flow-perpendicular size, the median near-magnetopause size of $0.93 R_{\mathrm{E}}$ [Plaschke et al., 2016] would correspond to $\sim 97 d_{\mathrm{i}}$ in our event.

\subsection{Event Dynamics}

Figure 4 summarizes the inferred dynamics of the event in the $L N$-plane of the THE inbound HMVA coordinates. (In this plane, the blue arrow indicating the local magnetopause normal vector is vertical when the magnetopause moves Sunward across THE in the fourth panel.) Note that the sketch does not capture the 3D nature of the event. For instance, THB was separated by $\sim 60 d_{\mathrm{i}}$ in the $M$ direction from the other spacecraft. 
At 22:56 UT, the magnetopause was moving Earthward over THB, and THC and $\mathrm{D}$ were located at its magnetospheric edge. This magnetopause inward motion does not appear to be driven by the HSJ studied here, since THB spent tens of seconds in the magnetosheath before it observed the jet. More likely it was due to surface waves excited by the previous HSJs during the preceding half an hour (as listed in Dmitriev and Suvorova [2015]). The magnetopause motion seen here $\left(\sim 1 R_{\mathrm{E}}\right)$ is comparable to the typical amplitude of magnetopause oscillations $\left(0.5-0.8 R_{\mathrm{E}}\right.$; e.g., Song et al. [1988]; Plaschke et al. [2009b]).

Two minutes later, at 22:58 UT, the magnetopause had moved over THE, while THC and D were at its magnetosheath edge. THB began to observe the magnetosheath HSJ. At 22:58:30 UT, THE was at the magnetosheath edge of the magnetopause, THB stopped seeing the HSJ while THC observed the HSJ-like density increase.

We can estimate that at a speed of $\sim 250 \mathrm{~km} / \mathrm{s} \sim 4 d_{\mathrm{i}} / \mathrm{s}$ towards the magnetopause, the HSJ would cover a distance of $\sim 120 d_{\mathrm{i}}$ in these $30 \mathrm{~s}$ (22:58:00-22:58:30). Therefore the jet had more than sufficient time to reach the magnetopause. The large increase in magnetosheath total pressure during the jet was likely sufficient to significantly compress the magnetopause boundary layer, at least momentarily, such that reconnection was be triggered. Given the jet's direction and expected first point of impact (Figure 4), the $\mathrm{X}$-point would form in the $+L$ direction from the spacecraft, resulting in a $-V_{L}$ outflow.

At 22:59:10 UT the magnetopause was moving outwards again, THE was on its magnetospheric edge and THC and D observed a reconnection outflow in the $-L$ direction. At 22:59:50 UT the magnetopause continued moving outwards across THB, which observed a fully formed reconnection outflow.

\section{Discussion and Conclusions}

We have examined THEMIS observations of a magnetosheath high-speed jet impact event, where the pre-impact magnetopause was thick $\left(60-70 d_{\mathrm{i}}\right)$, high shear $\left(140-160^{\circ}\right)$, and showed no signatures of magnetic reconnection. After the magnetosheath jet three spacecraft observed reconnection outflows within the magnetopause layer. Given that the magnetic shear did not change significantly from pre to post-impact magnetopause crossings, we think it is likely that reconnection was initiated by the jet's high dynamic pressure impact compressing the current layer (see scenario (i) in the Introduction). These observations constitute the first in situ evidence suggesting that HSJ impacts may trigger magnetopause reconnection.

OMNI solar wind data, propagated algorithmically from L1 to Earth, are commonly used to give context to magnetospheric dynamics, especially in statistical studies. There is naturally uncertainty related to propagation. For the event studies here, the OMNI IMF was northward during the impact while the magnetosheath magnetic field observed by THEMIS was southward (possibly due to magnetic draping; Figure 1(a,d,g,j)). Therefore the magnetic shear at the magnetopause was actually favorable for reconnection. However, the pre-impact magnetopause thickness seems to have been too large for reconnection to start.

The magnetopause thickness during the (non-reconnecting, pre-impact) outbound crossing was $60-70 d_{\mathrm{i}}(\sim 4000 \mathrm{~km})$, which is much thicker than usual $(100-3000 \mathrm{~km}$; e.g., Berchem and Russell [1982]; Phan and Paschmann [1996]). The reason for this is unclear. It may be related to the effects of previous HSJ impacts during the half an hour leading to the impact studied here [Dmitriev and Suvorova, 2015]. It may also be related to heavy and/or cold magnetospheric ions: We can see signatures of oxygen (a band around $1 \mathrm{keV}$ ) in the ion spectrograms of THC and D before the outbound crossing (Figure 2(c); distinguishable by their energy separation from the protons, e.g., Lee et al. [2012]). While there are no signatures of cold ions at THB, C, D, or E, they are present at the innermost probe 
THA ( $\sim 2 R_{\mathrm{E}}$ Earthwards from THE), evidenced by the rise and fall in their peak energy as the flow velocity increases-then-decreases (not shown).

It is interesting that there is no evident reconnection outflow at THE, although the shape of the $V_{L}$ curve is somewhat similar to THC (Figure 3(f)). Given that the magnetic shear at THE location was only $\sim 100^{\circ}$, reconnection may be suppressed by diamagnetic drift according to the $\Delta \beta$-shear relation and the observed boundary conditions [Swisdak et al., 2010; Phan et al., 2013]. The absence of outflow could also be related to the transient nature of the reconnection onset being triggered, or be a 3D effect.

The high occurrence rate of HSJ impacts suggests that by triggering bursts of magnetopause reconnection, the jets could make a significant contribution to the production of new open magnetic flux, especially during otherwise 'quiet' quasi-radial IMF conditions. Furthermore, localized, transient reconnection driven by the HSJs could at least modulate the global reconnection rate even if on average it may remain dictated by the upstream IMF. Future studies that address reconnection in association with magnetosheath jets in a systematic manner are required to determine how often it occurs. Due to the impacts reconnection could be either turned on or off, or stay unaffected; therefore, a statistical approach should be applied to quantify the overall effects for the solar wind-magnetosphere coupling. In addition, (global) kinetic simulations would help shed light on the details of the magnetopause reconnection initiated by the magnetosheath jets.

\section{Acknowledgments}

We acknowledge valuable discussions within the International Space Science Institute (ISSI) team 350 "Jets downstream of collisionless shocks" led by FP and HH. HH thanks R. Mistry, M.A. Shay and J.P. Eastwood for discussions, comments, and suggestions. The work of HH was supported by NASA grant NNX17AI45G and contract NAS5-02099. The work of FP was supported by Austrian FWF grant P 28764-N27. We thank the THEMIS software team and NASA's Coordinated Data Analysis Web (http://cdaweb.gsfc.nasa.gov/) for their analysis tools and data access. The THEMIS data and analysis software (SPEDAS) are freely available from http://themis.ssl.berkeley.edu. The version of SPEDAS and the routines used to make Figures 1, 2, 3, and S1 are provided as part of the article Supplementary material.

\section{References}

Amata, E., S. P. Savin, D. Ambrosino, Y. V. Bogdanova, M. F. Marcucci, S. Romanov, and A. Skalsky (2011), High kinetic energy density jets in the Earth's magnetosheath: A case study, Planet. Space Sci., 59, 482-494, doi:10.1016/j.pss.2010.07.021.

Angelopoulos, V. (2008), The THEMIS mission, Space Sci. Rev., 141, 5-34, doi: 10.1007/s11214-008-9336-1.

Archer, M. O., and T. S. Horbury (2013), Magnetosheath dynamic pressure enhancements: Occurrence and typical properties, Ann. Geophys., 31, 319-331, doi:10.5194/angeo-31319-2013.

Archer, M. O., T. S. Horbury, and J. P. Eastwood (2012), Magnetosheath pressure pulses: Generation downstream of the bow shock from solar wind discontinuities, J. Geophys. Res., 117, A05,228, doi:10.1029/2011JA017468.

Archer, M. O., M. D. Hartinger, and T. S. Horbury (2013a), Magnetospheric "magic" frequencies as magnetopause surface eigenmodes, Geophys. Res. Lett., 40, 5003-5008, doi: 10.1002/grl.50979.

Archer, M. O., T. S. Horbury, J. P. Eastwood, J. M. Weygand, and T. K. Yeoman (2013b), Magnetospheric response to magnetosheath pressure pulses: A low-pass filter effect, $J$. Geophys. Res., 118, 5454-5466, doi:10.1002/jgra.50519.

Auster, H. U., K. H. Glassmeier, W. Magnes, O. Aydogar, W. Baumjohann, D. Constantinescu, D. Fischer, K. H. Fornacon, E. Georgescu, P. Harvey, O. Hillenmaier, R. Kroth, 
M. Ludlam, Y. Narita, R. Nakamura, K. Okrafka, F. Plaschke, I. Richter, H. Schwarzl, B. Stoll, A. Valavanoglou, and M. Wiedemann (2008), The THEMIS Fluxgate Magnetometer, Space Sci. Rev., 141, 235-264, doi:10.1007/s11214-008-9365-9.

Berchem, J., and C. T. Russell (1982), The thickness of the magnetopause current layer: Isee 1 and 2 observations, J. Geophys. Res., 87(A4), 2108-2114, doi: 10.1029/JA087iA04p02108.

Blanco-Cano, X., N. Omidi, and C. T. Russell (2009), Global hybrid simulations: Foreshock waves and cavitons under radial interplanetary magnetic field geometry, J. Geophys. Res., 114, A01,216, doi:10.1029/2008JA013406.

Bonnell, J. W., F. S. Mozer, G. T. Delory, A. J. Hull, R. E. Ergun, C. M. Cully, V. Angelopoulos, and P. R. Harvey (2008), The Electric Field Instrument (EFI) for THEMIS, Space Sci. Rev., 141, 303-341, doi:10.1007/s11214-008-9469-2.

Cassak, P. A., and S. A. Fuselier (2016), Reconnection at Earth's Dayside Magnetopause, in Magnetic Reconnection: Concepts and Applications, Astrophysics and Space Science Library, vol. 427, edited by W. Gonzalez and E. Parker, pp. 213-217.

Cassak, P. A., and M. A. Shay (2007), Scaling of asymmetric magnetic reconnection: General theory and collisional simulations, Physics of Plasmas, 14(10), 102114, doi: 10.1063/1.2795630.

Dmitriev, A. V., and A. V. Suvorova (2012), Traveling magnetopause distortion related to a large-scale magnetosheath plasma jet: THEMIS and ground-based observations, $J$. Geophys. Res., 117, A08,217, doi:10.1029/2011JA016861.

Dmitriev, A. V., and A. V. Suvorova (2015), Large-scale jets in the magnetosheath and plasma penetration across the magnetopause: THEMIS observations, J. Geophys. Res., 120, 4423-4437, doi:10.1002/2014JA020953.

Elkington, S. R., M. K. Hudson, and A. A. Chan (2003), Resonant acceleration and diffusion of outer zone electrons in an asymmetric geomagnetic field, J. Geophys. Res., 108, 1116, doi:10.1029/2001JA009202.

Gosling, J. T., and T. D. Phan (2013), Magnetic Reconnection in the Solar Wind at Current Sheets Associated with Extremely Small Field Shear Angles, Astrophys. J. Lett., 763, L39, doi:10.1088/2041-8205/763/2/L39.

Gosling, J. T., M. F. Thomsen, S. J. Bame, R. C. Elphic, and C. T. Russell (1990), Cold ion beams in the low latitude boundary layer during accelerated flow events, Geophys. Res. Lett., 17, 2245-2248, doi:10.1029/GL017i012p02245.

Gunell, H., H. Nilsson, G. Stenberg, M. Hamrin, T. Karlsson, R. Maggiolo, M. André, R. Lundin, and I. Dandouras (2012), Plasma penetration of the dayside magnetopause, Phys. Plasmas, 19(7), 072906, doi:10.1063/1.4739446.

Gunell, H., G. Stenberg Wieser, M. Mella, R. Maggiolo, H. Nilsson, F. Darrouzet, M. Hamrin, T. Karlsson, N. Brenning, J. De Keyser, M. André, and I. Dandouras (2014), Waves in high-speed plasmoids in the magnetosheath and at the magnetopause, Ann. Geophys., 32, 991-1009, doi:10.5194/angeo-32-991-2014.

Han, D.-S., H. Hietala, X.-C. Chen, Y. Nishimura, L. R. Lyons, J.-J. Liu, H.-Q. Hu, and H.-G. Yang (2017), Observational properties of dayside throat aurora and implications on the possible generation mechanisms, J. Geophys. Res., 122(2), 1853-1870, doi: 10.1002/2016JA023394.

Hao, Y., B. Lembege, Q. Lu, and F. Guo (2016), Formation of downstream high-speed jets by a rippled nonstationary quasi-parallel shock: 2-D hybrid simulations, J. Geophys. Res., 121, 2080-2094, doi:10.1002/2015JA021419.

Hietala, H., and F. Plaschke (2013), On the generation of magnetosheath high-speed jets by bow shock ripples, J. Geophys. Res., 118, 7237-7245, doi:10.1002/2013JA019172.

Hietala, H., T. V. Laitinen, K. Andréeová, R. Vainio, A. Vaivads, M. Palmroth, T. I. Pulkkinen, H. E. J. Koskinen, E. A. Lucek, and H. Rème (2009), Supermagnetosonic Jets behind a Collisionless Quasiparallel Shock, Phys. Rev. Lett., 103, 245,001, doi: 10.1103/PhysRevLett.103.245001. 
Hietala, H., N. Partamies, T. V. Laitinen, L. B. N. Clausen, G. Facskó, A. Vaivads, H. E. J. Koskinen, I. Dandouras, H. Rème, and E. A. Lucek (2012), Supermagnetosonic subsolar magnetosheath jets and their effects: from the solar wind to the ionospheric convection, Ann. Geophys., 30, 33-48, doi:10.5194/angeo-30-33-2012.

Karimabadi, H., V. Roytershteyn, H. Vu, Y. Omelchenko, J. Scudder, W. Daughton, A. Dimmock, K. Nykyri, M. Wan, D. Sibeck, et al. (2014), The link between shocks, turbulence, and magnetic reconnection in collisionless plasmas, Phys. Plasmas, 21(6), 062,308, doi:10.1063/1.4882875.

Karlsson, T., N. Brenning, H. Nilsson, J.-G. Trotignon, X. Vallières, and G. Facsko (2012), Localized density enhancements in the magnetosheath: Three-dimensional morphology and possible importance for impulsive penetration, J. Geophys. Res., 117, A03227, doi: 10.1029/2011JA017059.

Khrabrov, A. V., and B. U. Ö. Sonnerup (1998), Orientation and motion of current layers: minimization of the Faraday residue, Geophys. Res. Lett., 25, 2373-2376, doi: 10.1029/98GL51784.

Lee, J. H., L. Chen, V. Angelopoulos, and R. M. Thorne (2012), Themis observations and modeling of multiple ion species and emic waves: Implications for a vanishing he+ stop band, J. Geophys. Res., 117(A6), doi:10.1029/2012JA017539, a06204.

Lin, Y., L. C. Lee, , and M. Yan (1996a), Generation of dynamic pressure pulses downstream of the bow shock by variations in the interplanetary magnetic field orientation, $J$. Geophys. Res., 101, 479-493, doi:10.1029/95JA02985.

Lin, Y., D. W. Swift, , and L. C. Lee (1996b), Simulation of pressure pulses in the bow shock and magnetosheath driven by variations in interplanetary magnetic field direction, J. Geophys. Res., 101, 27,251-27,269, doi:10.1029/96JA02733.

McFadden, J. P., C. W. Carlson, D. Larson, M. Ludlam, R. Abiad, B. Elliott, P. Turin, M. Marckwordt, and V. Angelopoulos (2008), The THEMIS ESA Plasma Instrument and In-flight Calibration, Space Sci. Rev., 141, 277-302, doi:10.1007/s11214-008-94402.

Nemecek, Z., J. Safrankova, L. Prech, D. G. Sibeck, S. Kokubun, and T. Mukai (1998), Transient flux enhancements in the magnetosheath, Geophys. Res. Lett., 25, 1273-1276, doi:10.1029/98GL50873.

Omidi, N., J. Berchem, D. Sibeck, and H. Zhang (2016), Impacts of spontaneous hot flow anomalies on the magnetosheath and magnetopause, J. Geophys. Res., 121, 3155-3169, doi:10.1002/2015JA022170.

Paschmann, G., I. Papamastorakis, W. Baumjohann, N. Sckopke, C. W. Carlson, B. U. Ö. Sonnerup, and H. Lühr (1986), The magnetopause for large magnetic shear: Ampte/irm observations, Journal of Geophysical Research: Space Physics, 91(A10), 11,099-11,115, doi:10.1029/JA091iA10p11099.

Phan, T. D., and G. Paschmann (1996), Low-latitude dayside magnetopause and boundary layer for high magnetic shear: 1. structure and motion, J. Geophys. Res., 101(A4), 7801-7815, doi:10.1029/95JA03752.

Phan, T. D., G. Paschmann, and B. U. Ö. Sonnerup (1996), Low-latitude dayside magnetopause and boundary layer for high magnetic shear: 2. occurrence of magnetic reconnection, Journal of Geophysical Research: Space Physics, 101(A4), 7817-7828, doi: 10.1029/95JA03751.

Phan, T. D., M. A. Shay, J. T. Gosling, M. Fujimoto, J. F. Drake, G. Paschmann, M. Oieroset, J. P. Eastwood, and V. Angelopoulos (2013), Electron bulk heating in magnetic reconnection at Earth's magnetopause: Dependence on the inflow Alfvén speed and magnetic shear, Geophys. Res. Lett., 40, 4475-4480, doi:10.1002/grl.50917.

Phan, T. D., M. A. Shay, C. C. Haggerty, J. T. Gosling, J. P. Eastwood, M. Fujimoto, K. Malakit, F. S. Mozer, P. A. Cassak, M. Oieroset, and V. Angelopoulos (2016), Ion Larmor radius effects near a reconnection $\mathrm{X}$ line at the magnetopause: THEMIS observations and simulation comparison, Geophys. Res. Lett., 43, 8844-8852, doi: 10.1002/2016GL070224. 
Plaschke, F., K.-H. Glassmeier, D. G. Sibeck, H. U. Auster, O. D. Constantinescu, V. Angelopoulos, and W. Magnes (2009a), Magnetopause surface oscillation frequencies at different solar wind conditions, Ann. Geophys., 27, 4521-4532, doi:10.5194/angeo-274521-2009.

Plaschke, F., K.-H. Glassmeier, H. U. Auster, V. Angelopoulos, O. D. Constantinescu, K.H. Fornaçon, E. Georgescu, W. Magnes, J. P. McFadden, and R. Nakamura (2009b), Statistical study of the magnetopause motion: First results from THEMIS, J. Geophys. Res., 114(A1), doi:10.1029/2008JA013423, a00C10.

Plaschke, F., H. Hietala, and V. Angelopoulos (2013), Anti-sunward high-speed jets in the subsolar magnetosheath, Ann. Geophys., 31, 1877-1889, doi:10.5194/angeo-31-18772013.

Plaschke, F., H. Hietala, V. Angelopoulos, and R. Nakamura (2016), Geoeffective jets impacting the magnetopause are very common, J. Geophys. Res., 121, 3240-3253, doi: 10.1002/2016JA022534.

Savin, S., E. Amata, L. Zelenyi, V. Budaev, R. Treumann, E. Lucek, J. Safrankova, Z. Nemezek, Y. Khotyaintsev, M. Andre, J. Buechner, H. Alleyne, P. Song, J. Blecki, J. L. Rauch, S. Romanov, S. Klimov, and A. Skalsky (2008), High kinetic energy jets in the earth's magnetosheath: Implications for plasma dynamics and anomalous transport, JETP Lett., 87, 593-599, doi:10.1134/S0021364008110015.

Schwartz, S. J., and D. Burgess (1991), Quasi-parallel shocks - A patchwork of threedimensional structures, Geophys. Res. Lett., 18, 373-376, doi:10.1029/91GL00138.

Shue, J.-H., J.-K. Chao, P. Song, J. P. McFadden, . K. H. G. A. Suvorova, 1 V. Angelopoulos, , and F. Plaschke (2009), Anomalous magnetosheath flows and distorted subsolar magnetopause for radial interplanetary magnetic fields, Geophys. Res. Lett., 36, L18,112, doi:10.1029/2009GL039842.

Song, P., R. C. Elphic, and C. T. Russell (1988), Isee 1 \& 2 observations of the oscillating magnetopause, Geophys. Res. Lett., 15(8), 744-747, doi:10.1029/GL015i008p00744.

Sonnerup, B. U. O., and L. J. Cahill, Jr. (1967), Magnetopause Structure and Attitude from Explorer 12 Observations, J. Geophys. Res., 72, 171, doi: 10.1029/JZ072i001p00171.

Sonnerup, B. U. Ö., G. Paschmann, I. Papamastorakis, N. Sckopke, G. Haerendel, S. J. Bame, J. R. Asbridge, J. T. Gosling, and C. T. Russell (1981), Evidence for magnetic field reconnection at the earth's magnetopause, Journal of Geophysical Research: Space Physics, 86(A12), 10,049-10,067, doi:10.1029/JA086iA12p10049.

Swisdak, M., M. Opher, J. Drake, and F. A. Bibi (2010), The vector direction of the interstellar magnetic field outside the heliosphere, Astrophys. J., 710(2), 1769, doi: 10.1088/0004-637X/710/1769.

Turner, D. L., Y. Shprits, M. Hartinger, and V. Angelopoulos (2012), Explaining sudden losses of outer radiation belt electrons during geomagnetic storms, Nature Physics, 8, 208-212, doi:10.1038/nphys2185.

Vines, S. K., S. A. Fuselier, K. J. Trattner, S. M. Petrinec, and J. F. Drake (2015), Ion acceleration dependence on magnetic shear angle in dayside magnetopause reconnection, Journal of Geophysical Research: Space Physics, 120(9), 7255-7269, doi: 10.1002/2015JA021464, 2015JA021464. 


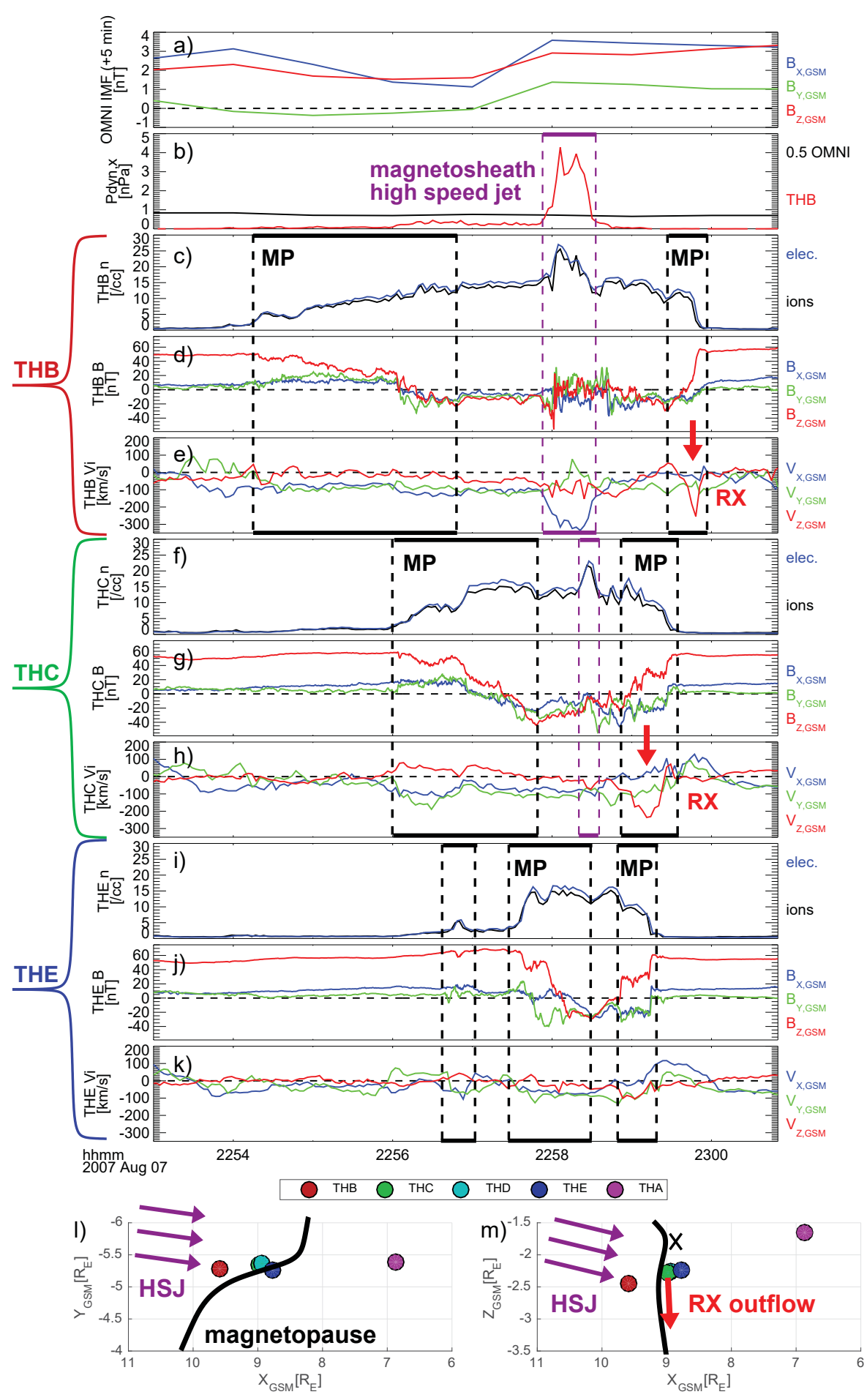

Figure 1. Overview of the event. (a) OMNI IMF, time-shifted by 5 minutes, (b) THB dynamic pressure in the $X$ direction (red) compared with half the OMNI dynamic pressure (black), (c) THB ion and electron densities, (d) THB magnetic field, (e) THB ion velocity, (f) THC ion and electron densities, (g) THC magnetic field, (h) THC ion velocity, (i) THE ion and electron densities, (j) THE magnetic field, (k) THE ion velocity. Panels (1) and (m) show the locations of the five THEMIS spacecraft in the $X Y_{\mathrm{GSM}}$ and $X Z_{\mathrm{GSM}}$ planes together with the high-speed jet direction (purple arrows), as well as a sketch of the magnetopause as it was moving outwards (black line), and of the reconnection outflow (red arrow). 
Outbound: no reconnection
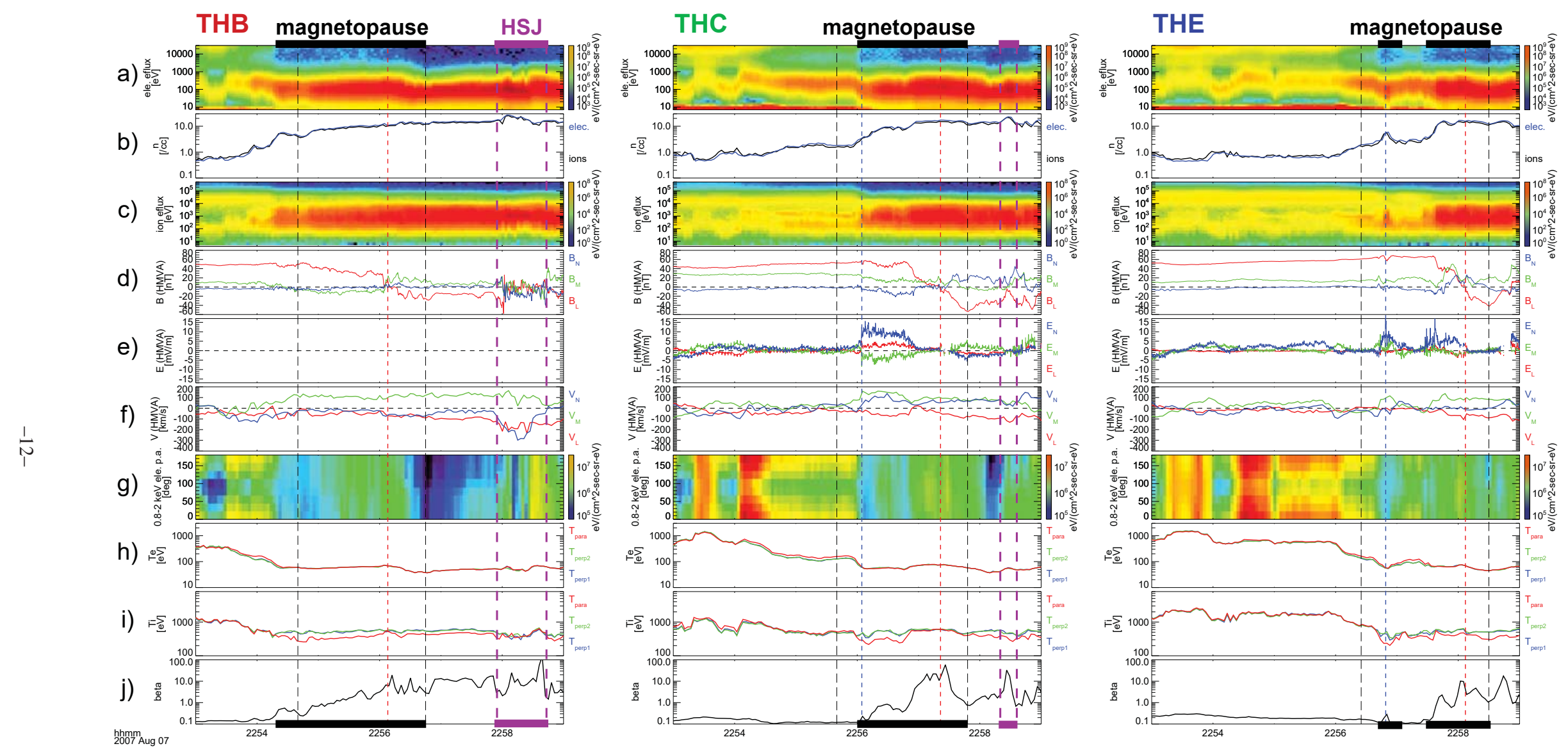

Figure 2. Outbound magnetopause crossing in the HMVA coordinates of each spacecraft. From left to right: THB, C, E. (a) electron energy spectrogram, (b) ion and electron densities, (c) ion energy spectrogram, (d) magnetic field, (e) electric field (no data for THB), (f) ion velocity, (g) 0.8-2 keV electron pitch-angle distribution, (h) electron temperature, (i) ion temperature, (j) total plasma beta. The black dashed lines indicate the interval used for HMVA analysis, the red dashed line marks where $B_{L} \quad=\quad 0$, and the blue dashed line marks the maximum of $E_{N}$ on the magnetospheric side. 


\section{Inbound: reconnection}
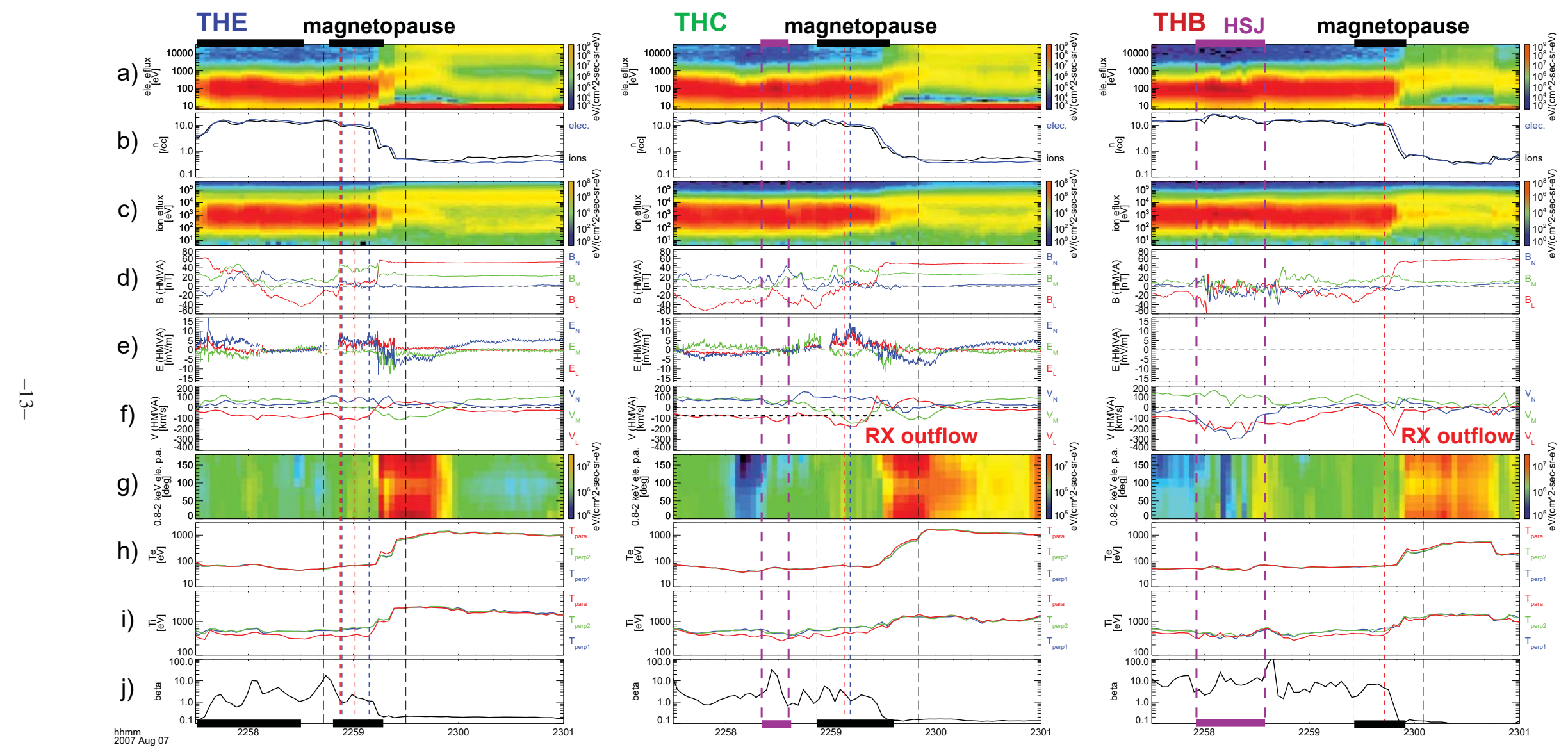

Figure 3. Inbound magnetopause crossing in the HMVA coordinates of each spacecraft. From left to right: THE, C, B. Same format as Figure 2. 

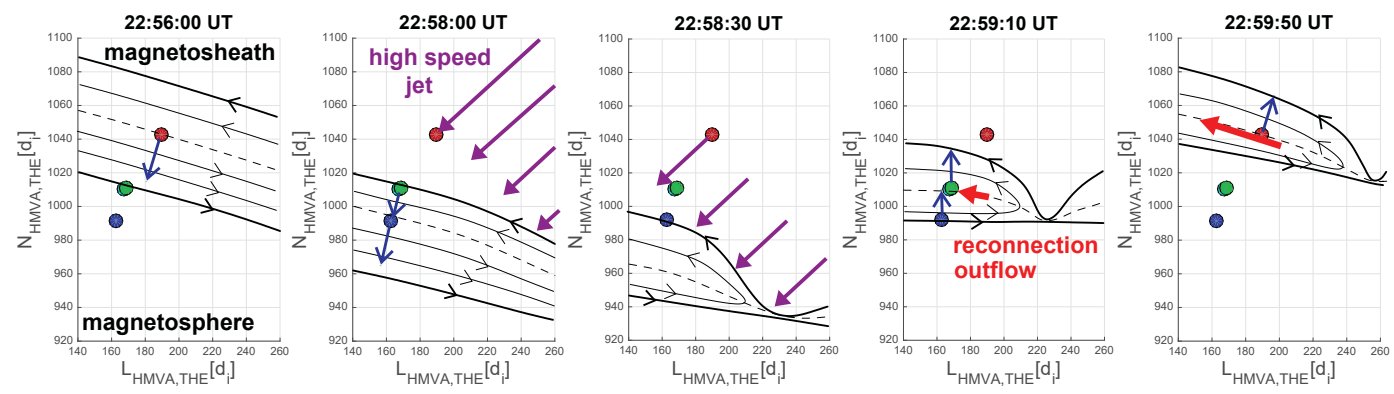

Figure 4. Series of sketches illustrating the high-speed jet impact and the evolution of the magnetopause reconnection. Black lines indicate the magnetopause layer, with a dashed line where $B_{L}=0$. The spacecraft (THB, C, D, and E; see Figure 1) and their normal vectors (blue arrows) are shown in the $L N_{\text {HMVA }}$ plane of the THE inbound crossing. I.e., the magnetopause is locally horizontal when it crosses THE in the fourth panel. Purple arrows show the high-speed jet and the red arrows the reconnection outflow. 
Figure 1. 


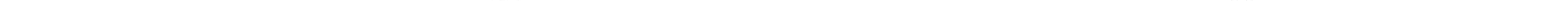


Figure 2. 


\section{Outbound: no reconnection}
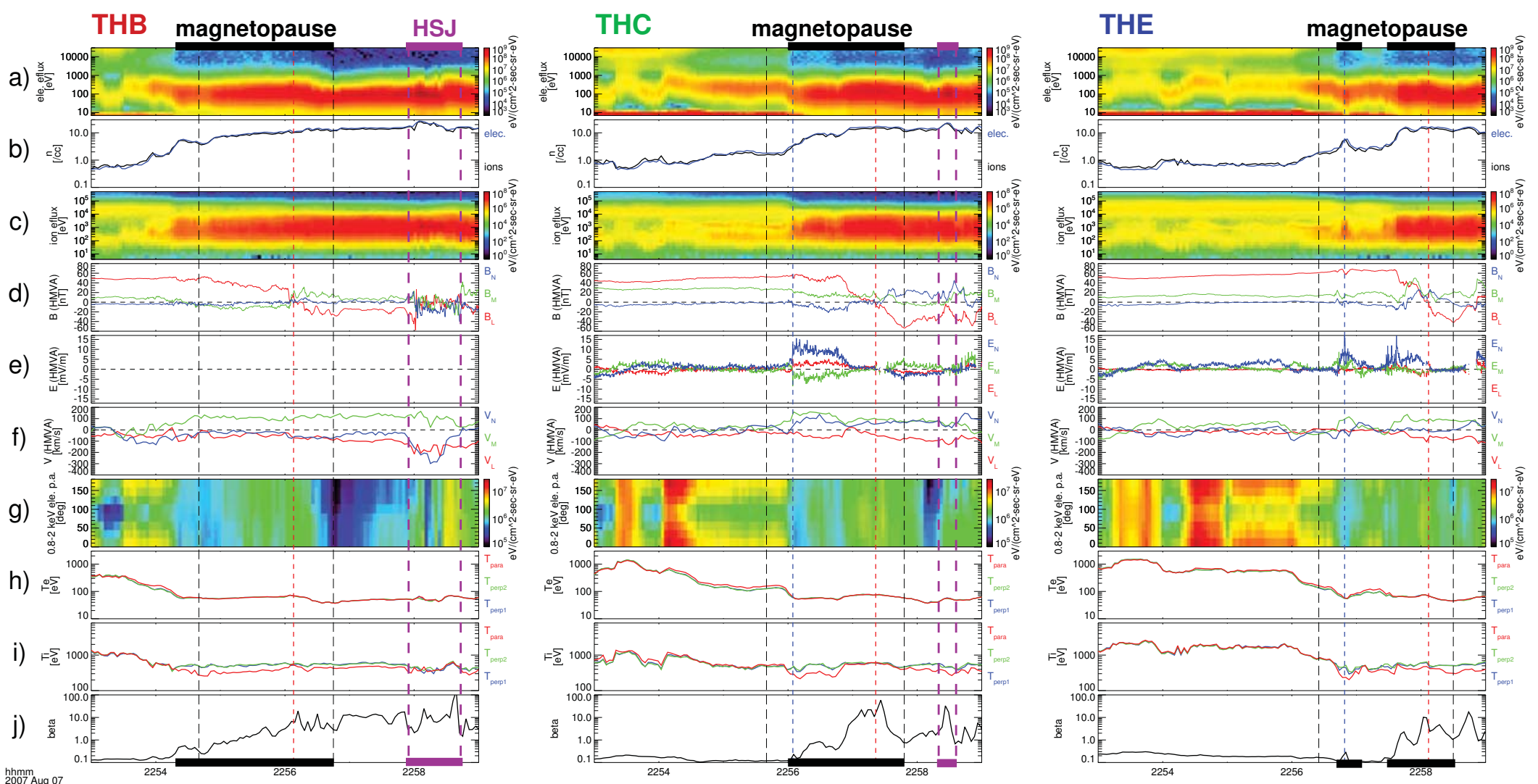
Figure 3. 


\section{Inbound: reconnection}
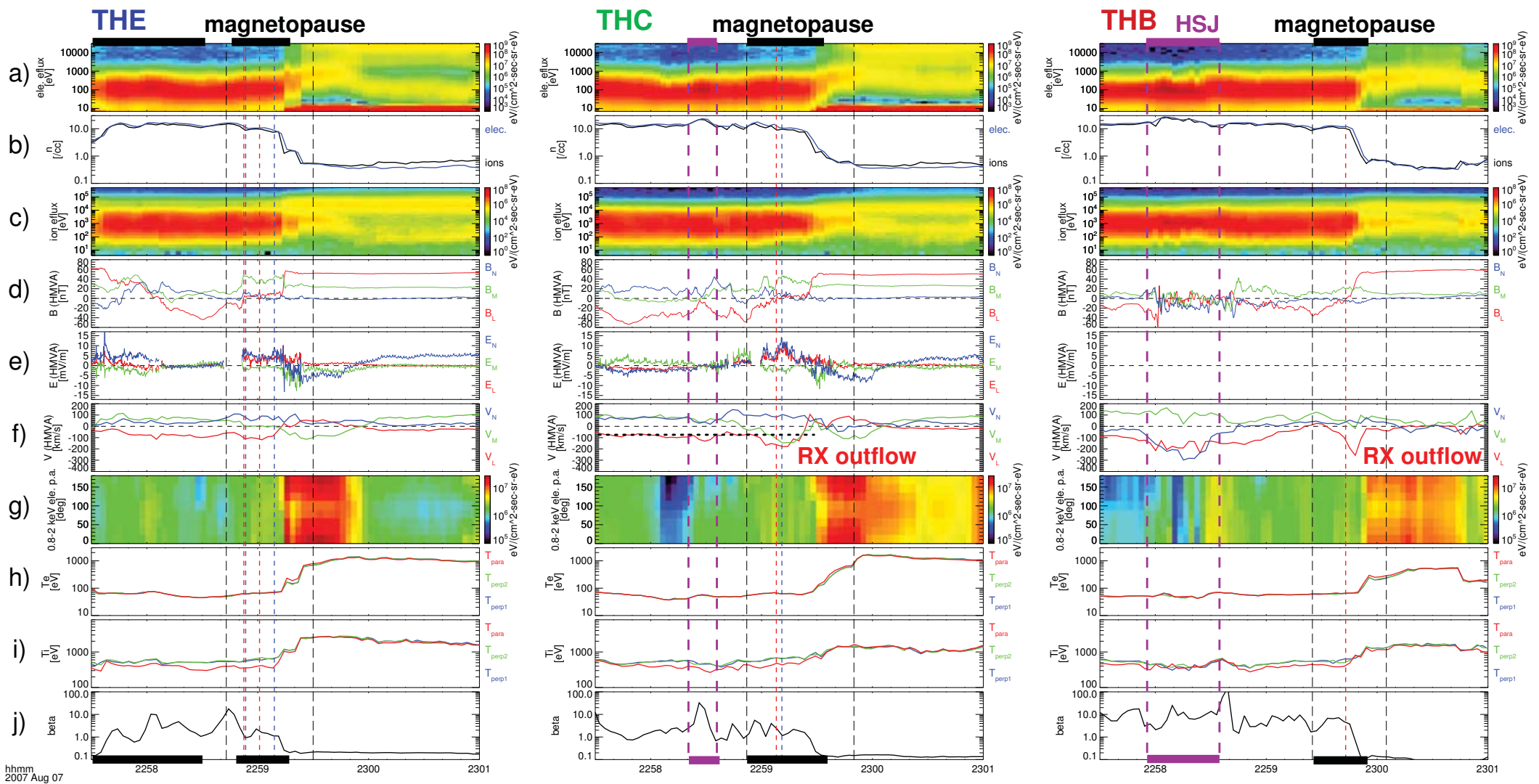
Figure 4. 

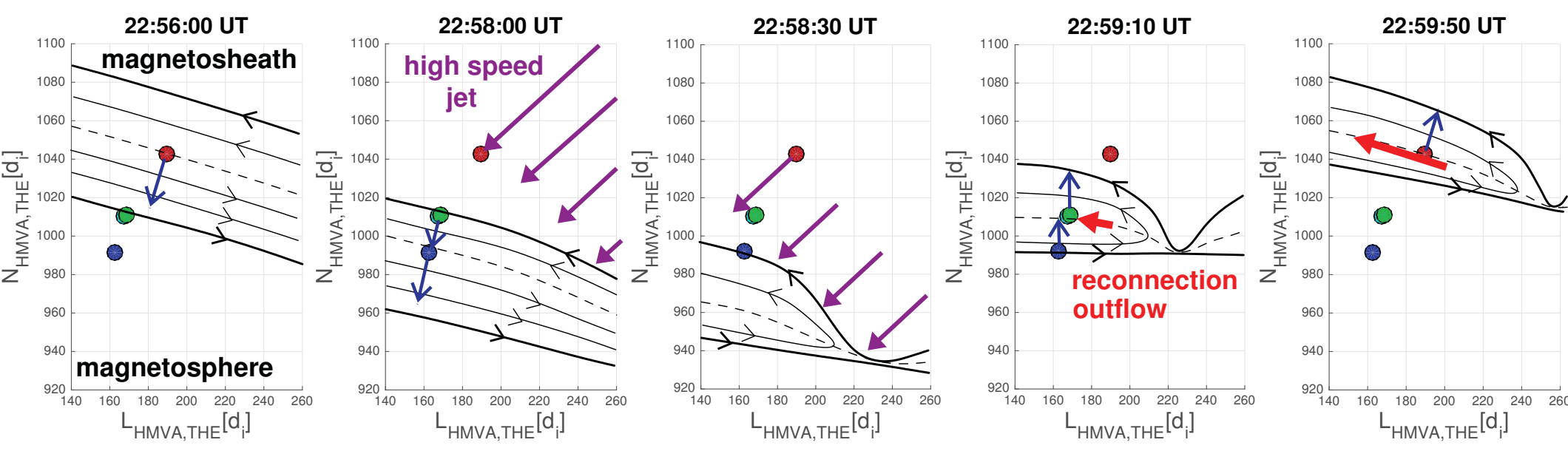